\title{
ELEMENTS IN EXCHANGE RINGS WITH RELATED COMPARABILITY
}

\author{
HUANYIN CHEN
}

(Received 23 December 1998)

\begin{abstract}
We show that if $R$ is an exchange ring, then the following are equivalent: (1) $R$ satisfies related comparability. (2) Given $a, b, d \in R$ with $a R+b R=d R$, there exists a related unit $w \in R$ such that $a+b t=d w$. (3) Given $a, b \in R$ with $a R=b R$, there exists a related unit $w \in R$ such that $a=b w$. Moreover, we investigate the dual problems for rings which are quasi-injective as right modules.
\end{abstract}

Keywords and phrases. Exchange ring, related comparability, related unit.

2000 Mathematics Subject Classification. Primary 16E50, 16L99.

Let $R$ be an associative ring with identity. From [6], $R$ is said to satisfy related comparability provided that for any idempotents $e, f \in R$ with $e=1+a b$ and $f=1+b a$ for some $a, b \in R$, there exists a $u \in B(R)$ such that $u e R \lessgtr^{\oplus} u f R$ and $(1-u) f R \lesssim^{\oplus}$ $(1-u) e R$. The class of rings satisfying related comparability is quite large. It includes regular rings satisfying general comparability [10], one-sided unit regular rings [8] and partially unit-regular rings, while there still exist rings satisfying related comparability, which belong to none of the above classes (cf., [7, Example 10]).

In $[4,5]$, we studied related comparability over regular rings. In [6, 7], we investigated related comparability over exchange rings. It is shown that every exchange ring satisfying related comparability is separative [1]. Also, we show that related comparability over exchange rings is a Morita invariant. $R$ is said to be an exchange ring if for every right $R$-module $A$ and any two decompositions $A=M \oplus N=\bigoplus_{i \in I} A_{i}$, where $M_{R} \cong R$ and the index set $I$ is finite, then there exist submodules $A_{i}^{\prime} \subseteq A_{i}$ such that $A=M \oplus\left(\bigoplus_{i \in I} A_{i}^{\prime}\right)$. Many authors have investigated exchange rings with some kind of comparability properties so as to study problems related partial cancellation properties of modules (see $[1,2,6,7,12,13]$ ).

In this paper, we investigate related comparability over exchange rings by related units. Recall that $w \in R$ is said to be a related unit of $R$ if there exists some $e \in B(R)$ such that $w=e u+(1-e) v$ for some $u, v \in R$, where $e u$ is right invertible in $e R$ and $(1-e) v$ is left invertible in $(1-e) R . w \in R$ is said to be a semi-related unit of $R$ if $w \in R$ is a related unit modulo $J(R)$. By virtue of semi-related units, we also give some new element-wise properties of rings which are quasi-injective as right modules.

Throughout, all rings are associative with identities. $B(R)$ denotes the set of all central idempotents of $R$ and $\mathrm{r} \cdot \operatorname{ann}(b)(\mathrm{l} \cdot \operatorname{ann}(b))$ denotes the right (left) annihilator of $b \in R$. 
LEMMA 1. Let $R$ be an exchange ring. Then $R$ satisfies related comparability if and only if so does the opposite ring $R^{\text {op }}$ of $R$.

Proof. Since $R$ is an exchange ring, by virtue of [11, Proposition], so is the opposite ring $R^{\mathrm{op}}$ of $R$. Assume that $R$ satisfies related comparability. Given $a^{\mathrm{op}}, b^{\mathrm{op}} \in R^{\mathrm{op}}$ with $a^{\mathrm{op}} x^{\mathrm{op}}+b^{\mathrm{op}}=1^{\mathrm{op}}$, then we have $x a+b=1$ in $R$. In view of [6, Theorem 4], there exists a $y \in R$ such that $x+b y$ is a related unit of $R$. Thus, we have some $e \in B(R)$ such that $(x+b y) e$ is right invertible in $e R$ and $(x+b y)(1-e)$ is left invertible in $(1-e) R$. By [5, Lemma 4], we claim that there are $z_{1}, z_{2} \in R$ such that $\left(a+z_{1} b\right) e$ is left invertible in $e R$ and $\left(a+z_{2} b\right)(1-e)$ is right invertible in $(1-e) R$. Let $z=z_{1} e+z_{2}(1-e)$. Then $a+z b$ is a related unit of $R$. Consequently, $a^{\mathrm{op}}+b^{\mathrm{op}} z^{\mathrm{op}}$ is a related unit of $R^{\mathrm{op}}$. By [6, Theorem 4], we conclude that $R^{\text {op }}$ satisfies related comparability. The converse is clear from $R \cong\left(R^{\mathrm{op}}\right)^{\mathrm{op}}$.

THEOREM 2. Let $R$ be an exchange ring. Then the following are equivalent:

(1) $R$ satisfies related comparability.

(2) Given $a, b, d \in R$ with $a R+b R=d R$, there exists a related unit $w \in R$ such that $a+b t=d w$.

(3) Given $a, b$ with $a R=b R$, there exists a related unit $w \in R$ such that $a=b w$.

(4) Given $a, b, d \in R$ with $R a+R b=R d$, there exists a related unit $w \in R$ such that $a+t b=w d$.

(5) Given $a, b$ with $R a=R b$, there exists a related unit $w \in R$ such that $a=w b$.

Proof. (2) $\Longrightarrow(1)$. Trivial from [6, Theorem 4].

$(1) \Longrightarrow(2)$. Given $a, b, d \in R$ with $a R+b R=d R$. Let $g: d R \rightarrow d R / b R$ be the canonical map, $f_{1}: R \rightarrow a R$ given by $r \mapsto a r$ for any $r \in R, f_{2}: R \rightarrow b R$ given by $r \mapsto b r$ for any $r \in R, f_{3}: R \rightarrow d R$ given by $r \mapsto d r$ for any $r \in R$. Since $a R+b R=d R$, we know that $g f_{1}, g f_{3}$ are epimorphisms. On the other hand, $R$ is a projective $R$-module. So there is some $\alpha \in \operatorname{End}_{R} R$ such that $g f_{1}=g f_{3} \alpha$. Since $g f_{1}$ is a epimorphism, we also have some $\psi \in \operatorname{End}_{R} R$ such that $g f_{3} \alpha \psi=g f_{3}$. From $\alpha \psi+(1-\alpha \psi)=1$, there is a $y \in \operatorname{End}_{R} R$ such that $\alpha+(1-\alpha \psi) y=w$ is a related unit of $\operatorname{End}_{R} R$. Therefore, we see that $g f_{1}=g f_{3} \alpha=g f_{3}(\alpha+(1-\alpha \psi) y)=g f_{3} w$, and then $g\left(f_{1}-f_{3} w\right)=0$. Thus, we have $\operatorname{Im}\left(f_{1}-f_{3} w\right) \leq \operatorname{Ker} g=b R$. By the projectivity of right $R$-module $R$, there exists some $\beta \in \operatorname{End}_{R} R$ such that $f_{2} \beta=f_{1}-f_{3} w$. Therefore, we claim that $a+b \beta(1)=f_{1}(1)+f_{2}(1) \beta(1)=f_{3}(1) w(1)=d w(1)$. It is easy to verify that $w(1)$ is a related unit of $R$.

$(1) \Longrightarrow(3)$. Given $a, b \in R$ with $a R=b R$, there exist $s, t \in R$ such that $a=b s$ and $b=a t$. Thus, $b=b s t$. Since $s t+(1-s t)=1$, by virtue of [6, Theorem 4 ], there exists some $z \in R$ such that $s+(1-s t) z=w$ is a related unit of $R$. Hence $a=b s=b(s+$ $(1-s t) z)=b w$, as desired.

(3) $\Longrightarrow(1)$. Given any regular $a \in R$. Then there exists some $b \in R$ such that $a=a b a$, so $a R=a b R$. Thus $a=a b w$ for some related unit $w \in R$. Since $a b+(1-a b)=1$, we see that $a+(1-a b) w=(a b+(1-a b)) w=w$. By [5, Lemma 4], there is some $z \in R$ such that $b+z(1-a b)=m$ is a related unit of $R$. Hence $a=a b a=a(b+z(1-a b)) a=$ ama. According to [6, Theorem 2], we claim that $R$ satisfies related comparability.

$(1) \Longleftrightarrow(4) \Longleftrightarrow(5)$. By [11, Proposition], we see that the opposite ring $R^{\text {op }}$ of $R$ is 
exchange. Using Lemma 1, we see that $R$ satisfies related comparability if and only if so does the opposite ring $R^{\text {op }}$ of $R$. Applying $(1) \Longleftrightarrow(2) \Longleftrightarrow(3)$. To $R^{\text {op }}$, we easily derive the result.

COROLLARY 3. Let $R$ be an exchange ring. Then the following are equivalent:

(1) $R$ satisfies related comparability.

(2) Given $a, b \in R$ with $a R+r \cdot \operatorname{ann}(b)=R$, there exists some $k \in \mathrm{r} \cdot \operatorname{ann}(b)$ such that $a+k$ is a related unit.

(3) Given $a, b \in R$ with $R a+1 \cdot \operatorname{ann}(b)=R$, there exists some $k \in 1 \cdot \operatorname{ann}(b)$ such that $a+k$ is a related unit.

PRoof. (1) $\Longrightarrow(2)$. Given $a, b \in R$ with $a R+\mathrm{r} \cdot \operatorname{ann}(b)=R$, then there exist $x \in R, k \in$ $\mathrm{r} \cdot \operatorname{ann}(b)$ such that $a x+k=1$. Since $R$ satisfies related comparability, by virtue of [6, Theorem 4], we can find a $y \in R$ such that $a+k y$ is a related unit of $R$. It is easy to check that $k y \in \mathrm{r} \cdot \operatorname{ann}(b)$, as required.

$(2) \Longrightarrow(1)$. Given $a, b \in R$ with $a R=b R$, there exist $s, t \in R$ such that $a=b s$ and $b=a t$. Obviously, $1-s t \in \mathrm{r} \cdot \operatorname{ann}(b)$. Since $s t+(1-s t)=1$, we have $s R+\mathrm{r} \cdot \operatorname{ann}(b)=R$. Thus we can find some $k \in \mathrm{r} \cdot \operatorname{ann}(b)$ such that $s+k=w$ is a related unit of $R$, and then $a=b s=b(s+k)=b w$, as asserted.

$(1) \Longleftrightarrow(3)$. Trivial by the symmetry of related comparability.

Recall that $n$ is in the stable range of $R$ provided that $a_{1} R+\cdots+a_{n+1} R=R$ with $a_{1}, \ldots, a_{n+1} \in R$ implies that $\left(a_{1}+a_{n+1} b_{1}\right) R+\cdots+\left(a_{n}+a_{n+1} b_{n}\right) R=R$ for some $b_{1}, \ldots, b_{n} \in R$. If no such $n$ exists, we say the stable range of $R$ is $\infty . x \in R$ is said to be related unit-regular if $x=x w x$ for some related unit $w \in R$. Now, we investigate related comparability by related unit-regularity as follows.

Proposition 4. Let $R$ be an exchange ring with the finite stable range. Then the following are equivalent:

(1) $R$ satisfies related comparability.

(2) Given $a, b, d \in R$ with $a R+b R=d R$, there exist some related unit-regular $w_{1}, w_{2} \in$ $R$ such that $a w_{1}+b w_{2}=d$.

(3) Given $a, b, d \in R$ with $R a+R b=R d$, there exist some related unit-regular $w_{1}, w_{2} \in$ $R$ such that $w_{1} a+w_{2} b=d$.

Proof. $(1) \Longrightarrow(2)$. Given $a R+b R=d R$ with $a, b, d \in R$. For right $R$-module $R^{2}$, the two sets $\{a, b\}$ and $\{0, d\}$ generate the same right $R$-submodule of $R^{2}$. Thus, we can find $A, B \in M_{2}(R)$ such that $(a, b)=(0, d) A,(0, d)=(a, b) B$. Assume that $A=\left(a_{i j}\right), B=\left(b_{i j}\right), I_{2}-A B=\left(c_{i j}\right) \in M_{2}(R)$. Since $A B+\left(I_{2}-A B\right)=I_{2}$, we have $\left(a_{21}, a_{22}\right)\left(b_{12}, b_{22}\right)^{T}+c_{22}=1$. Since $R$ is an exchange ring satisfying related comparability, its stable range can only be 1,2 or $\infty$ by [7, Theorem 3]. So 2 is in the stable range of $R$. Thus, we have some $\left(y_{1}, y_{2}\right) \in R^{2}$ such that $\left(a_{21}, a_{22}\right)+c_{22}\left(y_{1}, y_{2}\right) \in R^{2}$ is unimodular. Set $Y=\left(\begin{array}{cc}0 & 0 \\ y_{1} & y_{2}\end{array}\right)$. Then, we claim that the second row of $A+\left(I_{2}-A B\right) Y=U$ is unimodular. Clearly, $(0, d) U=(0, d) A=(a, b)$. Since $u_{21} R+u_{22} R=R$, we can find orthogonal idempotents $e_{1} \in u_{21} R, e_{2} \in u_{22} R$ such that $e_{1}+e_{2}=1$. Assume that $e_{1}=u_{21} x_{1}, e_{2}=u_{22} x_{2}$. Let $w_{1}=x_{1} e_{1}, w_{2}=x_{2} e_{2}$. Then $w_{1}$ and $w_{2}$ are both regular in $R$. Moreover, we have $u_{21} w_{1}+u_{22} w_{2}=1$. By the related comparability of $R$, we claim that both $w_{i}$ are related unit-regular, as asserted. 
$(2) \Longrightarrow(1)$. Given any regular $x \in R$. Then $x=x y x$ for a $y \in R$. So we have $x R+(1-$ $x y) R=R$, and then $x w_{1}+(1-x y) w_{2}=1$ for some related unit-regular $w_{1}, w_{2} \in R$. We easily check that $x+(1-x y) w_{2} s \in R$ is related unit for some $s \in R$. Hence $y+$ $t(1-x y)=w$, i.e., a related unit of $R$. Consequently, we show that $x=x y x=x w x$, as desired.

(1) $\Longleftrightarrow(3)$. Clear from the symmetry of related comparability.

Recall that a module $M$ is quasi-injective if any homomorphism of a submodule of $M$ into $M$ extends to an endomorphism of $M$. Now, we investigate rings which are quasi-injective as right modules. These extend the corresponding results in [3].

LEMMA 5. Let $R$ be quasi-injective as a right $R$-module. Given $a, b \in R$ with $a R+$ $b R=R$, there exists some $t \in R$ such that $a+b t$ is a semi-related unit.

Proof. Given $a, b \in R$ with $a R+b R=R$, then $\bar{a}(R / J(R))+\bar{b}(R / J(R))=R / J(R)$. Since $R$ is quasi-injective as a right $R$-module, by virtue of [9, Theorem 1$], R / J(R)$ is a regular, right self-injective ring. Hence $R$ is an exchange ring satisfying related comparability. According to Theorem 2, we can find a $y \in R$ such that $\bar{a}+\bar{b} \bar{y}=\bar{w}$ is a related unit of $R / J(R)$. Therefore $a+b y=w+r$ for some $r \in J(R)$. Clearly, $w+r$ is a semi-related unit of $R$, as desired.

THEOREM 6. Let $R$ be quasi-injective as a right $R$-module. Then the following hold:

(1) Given $a, b \in R$ with $\mathrm{r} \cdot \operatorname{ann}(a)=\mathrm{r} \cdot \operatorname{ann}(b)$, there exists a semi-related unit $w \in R$ such that $a=w b$.

(2) Given $a, b \in R$ with $\mathrm{l} \cdot \operatorname{ann}(a)=\mathrm{l} \cdot \operatorname{ann}(b)$, there exists a semi-related unit $w \in R$ such that $a=b w$.

Proof. (1) Given $a, b \in R$ with $\mathrm{r} \cdot \operatorname{ann}(a)=\mathrm{r} \cdot \operatorname{ann}(b)$. Since $R$ is quasi-injective as a right $R$-module, by [3, Lemma 3.2], we have $R a=R b$. Assume that $a=s b, b=t a$ for some $s, t \in R$. Then $b=t s b$. Consequently, there exists some $y \in R$ such that $t+(1-t s) y$ is a semi-related unit of $R$ by Lemma 5. Using [5, Lemma 4], we have some $z \in R$ such that $s+z(1-t s)=w$ is a semi-related unit of $R$. Therefore, we claim that $a=s b=(s+z(1-t s)) b=w b$, as desired.

(2) Given $a, b \in R$ with $1 \cdot \operatorname{ann}(a)=1 \cdot \operatorname{ann}(b)$. Similarly to the consideration above, we have $a R=b R$. Assume that $a=b s, b=a t$ for some $s, t \in R$. Then $b=b s t$. From $s t+(1-s t)=1$, we can find a $y \in R$ such that $s+(1-s t) y=w$ is a semi-related unit of $R$. Therefore $a=b s=b(s+(1-s t) y)=b w$, whence the result.

COROLlary 7. Let $R$ be quasi-injective as a left $R$-module. Then the following hold:

(1) Given $a, b \in R$ with $\mathrm{r} \cdot \operatorname{ann}(a)=\mathrm{r} \cdot \operatorname{ann}(b)$, there exists a semi-related unit $w \in R$ such that $a=w b$.

(2) Given $a, b \in R$ with $\mathrm{l} \cdot \operatorname{ann}(a)=1 \cdot \operatorname{ann}(b)$, there exists a semi-related unit $w \in R$ such that $a=b w$.

Proof. Applying Theorem 6 to the opposite ring $R^{\text {op }}$ of $R$, we complete the proof.

THEOREM 8. Let $R$ be a ring which is quasi-injective as a right $R$-module. Then the following hold: 
(1) Given $a, b \in R$ with $\mathrm{r} \cdot \operatorname{ann}(a) \cap \mathrm{r} \cdot \operatorname{ann}(b)=0$, there exists $t \in R$ such that $a+t b$ is a semi-related unit.

(2) Given $a, b \in R$ with $\mathrm{l} \cdot \operatorname{ann}(a) \cap \mathrm{l} \cdot \operatorname{ann}(b)=0$, there exists $t \in R$ such that $a+b t$ is a semi-related unit.

Proof. (1) Given $a, b \in R$ with $\mathrm{r} \cdot \operatorname{ann}(a) \cap \mathrm{r} \cdot \operatorname{ann}(b)=0$, by virtue of [3, Proposition 3.4], we know that $R a+R b=R$. Thus, $(R / J(R)) \bar{a}+(R / J(R)) \bar{b}=R / J(R)$. Since $R$ is a quasi-injective ring, from [9, Theorem 1$], R / J(R)$ is a regular, right self-injective ring. Moreover, we see that $R / J(R)$ satisfies related comparability. In view of Theorem 2 , there exists $t \in R$ such that $\bar{a}+\bar{t} \bar{b}=\bar{w}$ with $w$ is a semi-related unit of $R$. Thus, there is some $k \in J(R)$ such that $a+t b=w+k$. Clearly, $w+k$ is also a semi-related unit. Thus, we claim that $a+t b$ is a semi-related unit of $R$.

(2) Given $a, b \in R$ with $1 \cdot \operatorname{ann}(a) \cap 1 \cdot \operatorname{ann}(b)=0$, analogously to [3, Proposition 3.4], we claim that $a R+b R=R$. Thus $\bar{a}(R / J(R))+\bar{b}(R / J(R))=R / J(R)$. Similarly to the consideration above, we show that $R / J(R)$ satisfies related comparability. In view of Theorem 2, there exists $t \in R$ such that $a+b t=w+k$ with $w$ is a semi-related unit and $k \in J(R)$. Since $w+k$ is also a semi-related unit, the result follows.

COROLLARY 9. Let $R$ be a ring which is quasi-injective as a left $R$-module. Then the following hold:

(1) Given $a, b \in R$ with $\mathrm{r} \cdot \operatorname{ann}(a) \cap \mathrm{r} \cdot \operatorname{ann}(b)=0$, there exists $t \in R$ such that $a+t b$ is a semi-related unit.

(2) Given $a, b \in R$ with $\mathrm{l} \cdot \operatorname{ann}(a) \cap \mathrm{l} \cdot \operatorname{ann}(b)=0$, there exists $t \in R$ such that $a+b t$ is a semi-related unit.

Proof. Applying Theorem 8 to the opposite $\operatorname{ring} R^{\text {op }}$ of $R$, we easily obtain the result.

Since every regular, right (left) self-injective ring is a quasi-injective ring with trivial Jacobson. As an immediate consequence of Theorem 6, Corollary 7, Theorem 8, and Corollary 9, we now derive the following.

COROLLARY 10. Let $R$ be a regular, right (left) self-injective ring. Then the following hold:

(1) Given $a, b \in R$ with $\mathrm{r} \cdot \operatorname{ann}(a)=\mathrm{r} \cdot \operatorname{ann}(b)$, there exists a related unit $w \in R$ such that $a=w b$.

(2) Given $a, b \in R$ with $\mathrm{l} \cdot \operatorname{ann}(a)=1 \cdot \operatorname{ann}(b)$, there exists a related unit $w \in R$ such that $a=b w$.

(3) Given $a, b \in R$ with $\mathrm{r} \cdot \operatorname{ann}(a) \cap \mathrm{r} \cdot \operatorname{ann}(b)=0$, there exists $t \in R$ such that $a+t b$ is a related unit.

(4) Given $a, b \in R$ with $\mathrm{l} \cdot \operatorname{ann}(a) \cap \mathrm{l} \cdot \operatorname{ann}(b)=0$, there exists $t \in R$ such that $a+b t$ is a related unit.

\section{REFERENCES}

[1] P. Ara, K. R. Goodearl, K. C. O'Meara, and E. Pardo, Separative cancellation for projective modules over exchange rings, Israel J. Math. 105 (1998), 105-137. MR 99g:16006. Zbl 908.16002.

[2] V.P. Camillo and H. P. Yu, Stable range one for rings with many idempotents, Trans. Amer. Math. Soc. 347 (1995), no. 8, 3141-3147. MR 95j:16006. Zbl 848.16008. 
[3] M. J. Canfell, Completion of diagrams by automorphisms and Bass' first stable range condition, J. Algebra 176 (1995), no. 2, 480-503. MR 97a:16004. Zbl 839.16007.

[4] H. Chen, Related comparability over regular rings, Algebra Colloq. 3 (1996), no. 3, 277 282. MR 97j:16015. Zbl 857.16011.

[5] _ On related unit-regular elements, Algebra Colloq. 4 (1997), no. 3, 323-328. CMP 1681 549. Zbl 887.16009.

[6]_ Exchange rings, related comparability and power-substitution, Comm. Algebra 26 (1998), no. 10, 3383-3401. MR 99f:16005. Zbl 914.16001.

[7] __ Related comparability over exchange rings, Comm. Algebra 27 (1999), no. 9, 42094216. CMP 1705862.

[8] G. Ehrlich, Units and one-sided units in regular rings, Trans. Amer. Math. Soc. 216 (1976), 81-90. MR 52\#8183. Zbl 315.16008.

[9] K. R. Goodearl, Direct sum properties of quasi-injective modules, Bull. Amer. Math. Soc. 82 (1976), no. 1, 108-110. MR 53\#5665. Zbl 321.16016.

[10] _ von Neumann Regular Rings, Pitman (Advanced Publishing Program), Boston, Mass. London, 1979. MR 80e:16011. Zbl 411.16007.

[11] W. K. Nicholson, On exchange rings, Comm. Algebra 25 (1997), no. 6, 1917-1918. CMP 1446 139. Zbl 883.16003.

[12] E. Pardo, Comparability, separativity, and exchange rings, Comm. Algebra 24 (1996), no. 9, 2915-2929. MR 97k:16007. Zbl 859.16001.

[13] H. P. Yu, Stable range one for exchange rings, J. Pure Appl. Algebra 98 (1995), no. 1, 105-109. MR 96g:16006. Zbl 837.16009.

Chen: Department of Mathematics, Hunan Normal University, Changsha 410006, CHINA

E-mail address: chyzx1@sparc2.hunnu.edu.cn 


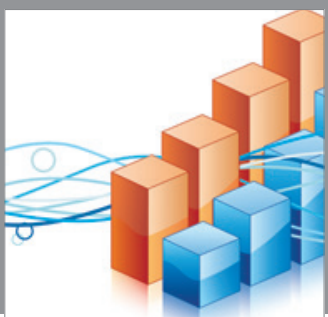

Advances in

Operations Research

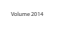

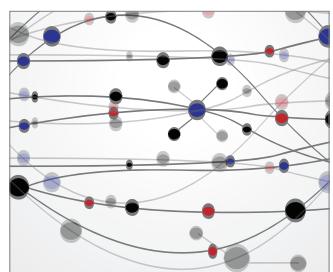

\section{The Scientific} World Journal
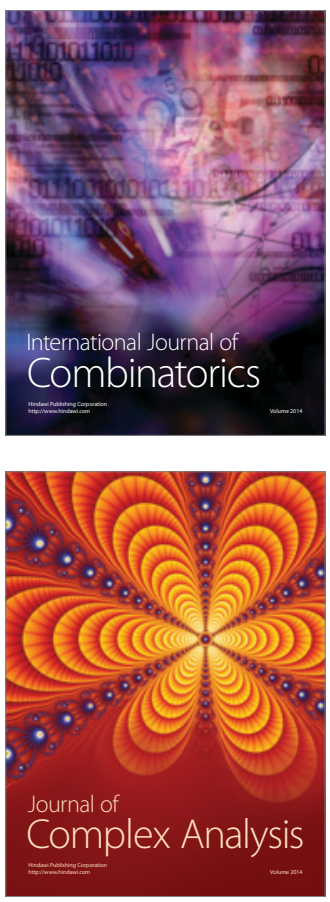

International Journal of

Mathematics and

Mathematical

Sciences
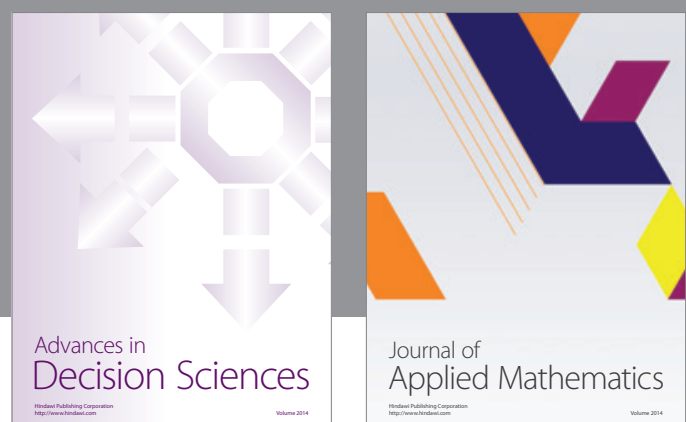

Journal of

Applied Mathematics
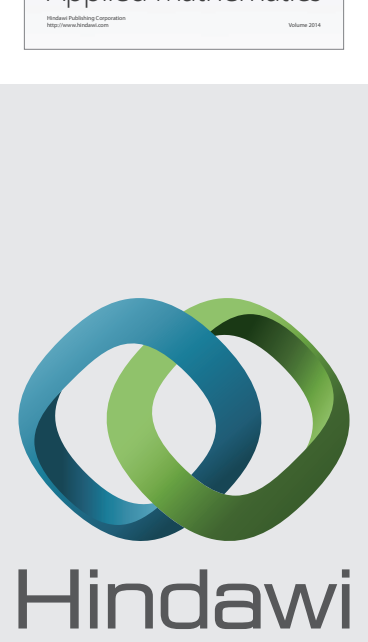

Submit your manuscripts at http://www.hindawi.com
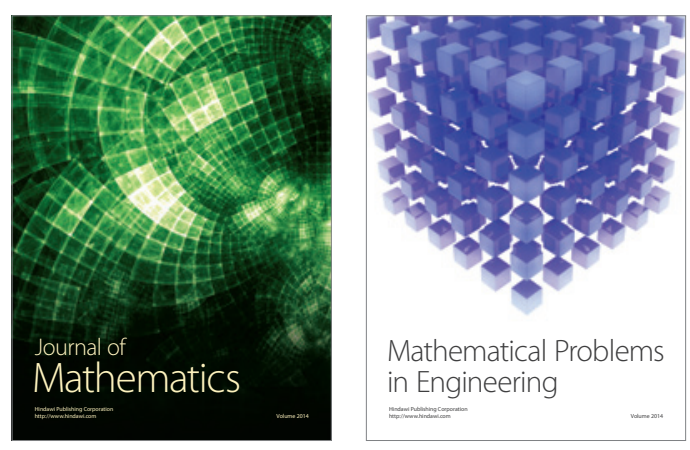

Mathematical Problems in Engineering
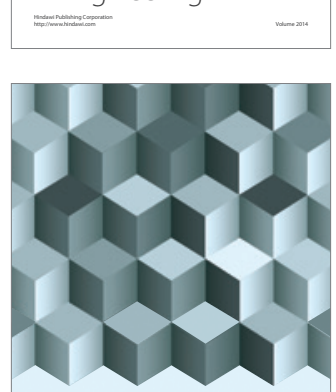

Journal of

Function Spaces
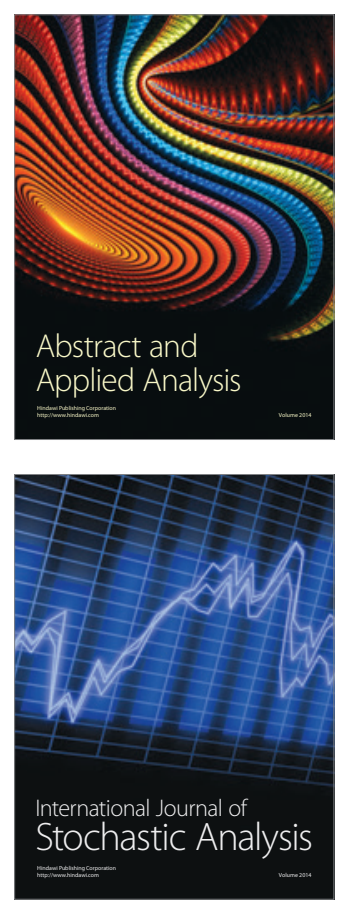

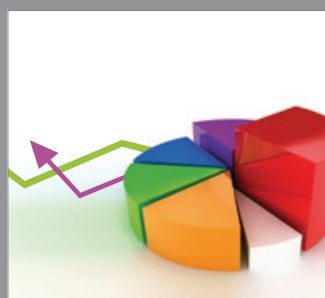

ournal of

Probability and Statistics

Promensencen
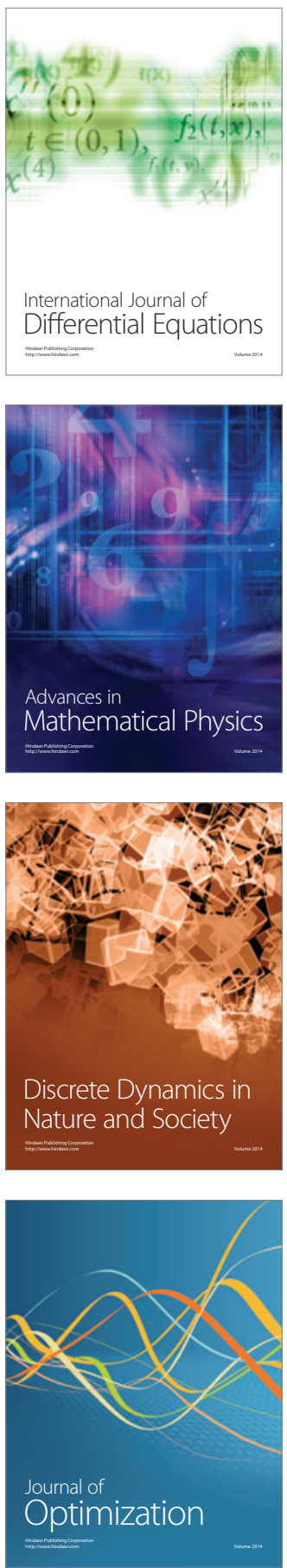